LBL-20650

\title{
Comet showers are not induced by interstellar clouds
}

\author{
Donald E. Morris \\ Lawrence Berkeley Laboratory \\ University of California, \\ Berkeley CA 94720
}

November 1985

"This work was prepared for the US Department of Energy under Contract Number DE-AC 03-76SF00098 


\title{
Comet showers are not induced by interstellar clouds
}

\author{
Donald E. Morris
}

\begin{abstract}
Encounters with interstellar clouds (IC) have been proposed by Rampino and Stothers ${ }^{1}$ as a cause of quasi-periodic intense comet showers leading to earth impacts, in order to explain the periodicity in marine mass extinctions found by Raup annd Sepkoski ${ }^{2}$ The model was described further ${ }^{3}$, criticised ${ }^{4-6}$ and defended. ${ }^{7}$ The debate has centered on the question of whether the scale height of the clouds is small enough (in comparison to the amplitude of the oscillation of the solar system about the plane of the Galaxy) to produce a modulation in the rate of encounters. We wish to point out another serious, we believe fatal, defect in this model -- the tidal fields of ICs are not strong enough to produce intense comet showers leading to earth impacts by bringing comets of the postulated inner Oort cloud into earth crossing orbits, except possibly during very rare encounters with very dense clouds. We will show that encounters with abundant clouds of low density cannot produce comet showers; cloud density $\mathrm{N}>10^{3}$ atoms $\mathrm{cm}^{-3}$ is needed to produce an intense comet shower leading to earth impacts. Furthermore, the tidal field of a dense cloud during a distant encounter is too weak to produce such showers. As a consequence, comet showers induced by ICs will be far less frequent than showers caused by passing stars. This conclusion is independent of assumptions about the radial distribution of comets in the inner Oort cloud.
\end{abstract}


We first review the conditions for bringing comets from the Oort cloud into earth crossing orbits. Because of perturbations by passing stars, comets with a given binding energy and semimajor axis $a$ will be found in orbits with all perihelia down to a limiting value $\approx 15$ AU which is determined by interactions with the major planets. 8,9 When the perihelion distance q of a comet falls below about $15 \mathrm{AU}$ it will gain or lose energy by interaction with Jupiter and Saturn. The interaction takes place near the perihelion of the large highly eccentric comet orbit $(\mathrm{q}<<a)$. The fractional change in velocity of the comet caused by the planets is small, so the fractional changes of angular momentum L and perihelion distance $\mathrm{q}$ are both small. However, near perihelion the kinetic and potential energy of the weakly bound comet are nearly equal but of opposite sign, and the mean change of energy caused by Jupiter and Saturn is larger than the very small binding energy of the comet. If energy is gained, the comet may escape from the solar system entirely, while if energy is lost, the new semimajor axis of the comet's orbit is reduced to a fraction of the previous value, but $\mathrm{q}$ is nearly unchanged. The comet is again perturbed by the major planets during its next perihelion passage and gains or loses energy randomly. This process continues until the comet is destroyed or ejected from the solar system. As a consequence, the solar system inside the orbit of Jupiter is screened off by the major planets unless a comet in a highly eccentric orbit with $\mathrm{q} \geq 15 \mathrm{AU}$ loses enough angular momentum during one orbit to bring it into an earth crossing orbit ( $\mathrm{q} \leq 1)$ at its next perihelion passage. We use the convenient units of solar mass $\mathrm{M}_{\mathrm{O}}$, length in $A U$ and time in yrs. In these units the gravitational constant $G=(2 \pi)^{2}$. The required change in specific angular momentum $\Delta \mathrm{L} / \mathrm{m} \geq 26 \mathrm{AU}^{2} / \mathrm{yr}$., since $\mathrm{L} / \mathrm{m} \approx 2 \pi(2 \mathrm{q})^{1 / 2}$ for a nearly parabolic orbit in these units. For orbits with $a \geq \mathrm{a}_{\mathrm{m}} \approx 2.5 \times 10^{4} \mathrm{AU}$ the screening is not effective since the tidal field of the Galaxy ${ }^{11}$ as well as numerous weak interactions with passing stars ${ }^{8}$ are strong enough to change the comet's specific angular momentum $\mathrm{L} / \mathrm{m}$ by more than the required amount in a single period $\mathrm{P}=a^{3 / 2}$ of the comet. As a result the earth is exposed at all times to a statistical sample of the comets which have $a>\mathrm{a}_{\mathrm{m}}$, and the flux of such comets will not be changed by a perturber.

Hills 12 suggested that the comets in the Oort cloud with $a \geq 2 \times 10^{4}$ represent only a "halo" containing $\approx 1 \%$ of the comets in a more massive "inner Oort cloud" consisting of comets with $3 \times 10^{3} \mathrm{AU} \leq a \leq 2 \times 10^{4} \mathrm{AU}$, and that the outer cloud comets have been supplied by scattering of inner cloud comets into the loss cone by relatively close stellar passages, followed by scattering by the major planets into higher energy orbits. He estimated the density of the inner cloud by several methods, and calculated that the close passage of a star at a distance $\approx 3 \times 10^{3}$ AU would produce a 
comet shower from the inner cloud so intense that several earth impacts would result. Hills ${ }^{13}$ calls this a "death shower".

In the presence of a static or slow moving perturber, the solar system comets move in a non-central gravitational field, so their angular momentum about the sun is not conserved. The rate of change of angular momentum of a comet is given by the torque from the tidal field of the perturber. The change of angular momentum $\mathrm{L}$ of a comet during time dt will be:

$$
\mathrm{d} \mathbf{L}=\mathrm{m} \Delta \mathbf{v} \times \mathbf{R}=\mathrm{m} x \mathrm{dA} / \mathrm{d} x \mathrm{dt} \sin \varnothing \mathrm{dt}=\mathrm{mdA} / \mathrm{dxd} \mathrm{d}^{2} \sin \varnothing \cos \varnothing \mathrm{dt}
$$

where the perturber is in the $\mathrm{x}$ direction and $\mathrm{dA} / \mathrm{dx}$ is its tidal acceleration, $\varnothing$ is the angle between the semimajor axis of the comet orbit the perturber, $m$ is the comet mass and $d$ is its distance from the sun. The tidal field inside a diffuse object such as a molecular cloud is $d A / d r=\left(32 \pi^{3} / 3\right) \rho$ in the approximation of a spherically symmetric cloud of uniform density, since the acceleration $\mathrm{A}=$ $\mathrm{G} \mathrm{M} / \mathrm{r}^{2}$, and the mass $\mathrm{M}=4 \pi / 3 \mathrm{r}^{3} \rho$, where $\mathrm{r}$ is the central distance. Outside the cloud $\mathrm{dA} / \mathrm{dr}=$ $\left(32 \pi^{3} / 3\right) \rho(\mathrm{R} / \mathrm{r})^{3}$ where $\mathrm{R}$ is the cloud radius. In more conventional units $\rho=2.8 \times 10^{-18} \mathrm{~N} \mathrm{M} O$ $\mathrm{AU}^{-3}$ where $\mathrm{N}$ is the number density of hydrogen atoms $/ \mathrm{cm}^{3}$, so $(\mathrm{dA} / \mathrm{dr})=9.3 \times 10^{-16} \mathrm{~N}$ inside the cloud.

The effective duration of interaction during a penetrating encounter $T \approx R / v$. So long as $T$ $<\mathrm{P}=a^{3 / 2}$ the comet period, we can use the impulse approximation in which we integrate $\mathrm{dL}$ over the time R/v. A large fraction of comets in highly eccentric orbits with a given $a$ will be found near aphelion at any given time, since a comet in a highly eccentric orbit is a distance $\mathrm{d} \geq a$ during $\approx 0.82$ of its period, and the time averaged value of $\mathrm{d}$ in this part of the orbit is $1.78 a{ }^{14}$ We use the approximations $\mathrm{d} \approx 1.78 a$, and $\sin \emptyset \cos \varnothing \approx 1 / 2$. A comet can be brought in from $\mathrm{q} \geq 15$ into an earth crossing orbit $(\mathrm{q} \leq 1)$ if $\Delta \mathrm{L} / \mathrm{m}>26$. Substituting in (1) we find

$$
\mathrm{a}_{\mathrm{S}} \approx 1.35 \times 10^{5}(\mathrm{v} / \mathrm{R} \mathrm{N})^{1 / 2} \mathrm{AU} \approx 6 \times 10^{5} \mathrm{R}^{-1 / 2} \mathrm{~N}^{-1 / 2} \mathrm{AU}
$$

where $R$ is in parsecs and $v$ in $\mathrm{km} / \mathrm{s}$, we take $\mathrm{v} \approx 20 \mathrm{~km} / \mathrm{s}$, the velocity of the sun with respect to the local standard of rest. 15 Then the condition $\mathrm{T}=\mathrm{R} / \mathrm{v}<\mathrm{a}_{\mathrm{S}}{ }^{3 / 2}$ is satisfied if $\mathrm{N}<1.8 \times 10^{5} \mathrm{R}^{-7 / 3}$ in these units. It may be noted that the value of $a_{s}$ during a close or penetrating encounter with an interstellar cloud depends directly on the cloud density rather than the cloud mass. 
Values of $\mathrm{a}_{\mathrm{S}}$ were calculated for each class of IC given in Table I of Ref 15 and for GMCs based on data from Ref 16 . The cumulative rate of encounters with the classes of IC which would reduce $a_{S}$ below a given value is given in Fig. 1 along with $\tau$ the mean interval between encounters. For comparison we give the rate of encounters and $\tau$ vs $a_{s}$ for comet showers induced by passing stars, after Hills. ${ }^{12}$ The abundant "standard" clouds which have typical density $\mathrm{N} \approx 10-30$ atoms $\mathrm{cm}^{-3}$ are encountered frequently $\left(\tau \approx 1-3 \times 10^{7} \mathrm{yrs}\right)$, but the calculated value of $\mathrm{a}_{\mathrm{S}} \approx 7 \times 10^{4} \mathrm{AU}$. Since the loss cone for comets in the Oort cloud with a $>2 \times 10^{4} \mathrm{AU}$ is kept filled by the Galactic field, encounters with "standard" clouds do not produce any increase in comet flux. In fact even an encounter with a cloud with $\mathrm{N} \approx 10^{2}$ (Lynds dark nebula class $\mathrm{j}=1$ ) would give $\mathrm{a}_{\mathrm{i}} \approx 2.4 \times 10^{4} \mathrm{AU}$ and would not produce a comet shower. Encounters with denser clouds (Lynds classes $\mathrm{j}=2$ to $\mathrm{j}=6$ ) with $2 \times 10^{2} \leq \mathrm{N} \leq 5 \times 10^{3}$ would reduce $\mathrm{a}_{\mathrm{S}}$ sufficiently to produce comet showers, but at very long intervals (see Fig 1). The plotted values of $\mathrm{a}_{\mathrm{S}}$ and $\tau$ for GMCs are based on mean density $\mathrm{N} \approx 290(\mathrm{R} / 10 \text { parsec })^{-0.75}$ atoms $\mathrm{cm}^{-3}$ and size $10 \leq \mathrm{R} \leq 40$ parsecs. ${ }^{16}$ The results are unchanged if the GMCs are taken to be "clumpy" with higher density subclouds $\left(\mathrm{R} \approx 2 \mathrm{pc}\right.$ and $\left.\mathrm{N} \approx 10^{3}\right) .17$

Stothers ${ }^{3}$ suggested that the relevent perturbers might be the dark matter in the disc of the Galaxy. If the dark matter is diffuse with a scale of several parsecs, then the analysis of this paper applies and the production of a death shower requires a density $\mathrm{N}>10^{3}$, or $25 \mathrm{M}_{\mathrm{O}} \mathrm{pc}^{-3}$.

The inequality $\mathrm{R} / \mathrm{v}<\mathrm{a}_{\mathrm{s}}{ }^{3 / 2}$ is not satisfied in an encounter with a large GMC $(\mathrm{R}>20 \mathrm{pc})$, so the effective duration of interaction is limited to one comet period. Then from Ref 11, the smallest comet orbits with filled loss cone have $a \approx \mathrm{a}_{\mathrm{m}}=2.18(\mathrm{dA} / \mathrm{dx})^{-2 / 7}=4.3 \times 10^{4} \mathrm{~N}^{-2 / 7}=10^{4}$ AU for a large GMC $(R \approx 30 \mathrm{pc})$. This result is nearly the same as for smaller clouds, where the impulse approximation is applicable. The volume fraction of GMCs $f \approx 0.08$ and mean free path $\approx 3.5 \times 10^{3}$ parsec in the inner galaxy, with half as many GMCs at the sun's galactic radius. ${ }^{16}$ This gives an encounter rate below one per $4 \times 10^{8}$ yrs. Thus, encounters with GMCs are not a significant cause of comet showers. The encounter rate $v s \mathrm{a}_{\mathrm{S}}$ of molecular clouds based on $\mathrm{CO}$ measurements indicated in Ref 15 also falls below the dashed line in Fig 1, but is not plotted since the estimates of cloud size and density are out of date.

Hills ${ }^{13}$ estimated that it is necessary to bring in comets with $a \approx 4000 \mathrm{AU}$, in order to produce a death shower. This requires a cloud density $\mathrm{N} \geq 10^{4}$ atoms $\mathrm{cm}^{-3}$ according to eq 2 , if $\mathrm{r} \approx 2 \mathrm{pc}$ and $\mathrm{v} \approx 20 \mathrm{~km} / \mathrm{s}$. Clouds of such high density are very rare; not even a single encounter 
would be expected over the life of the solar system according to Fig. 1 of Ref 15 . The density of a typical GMC is too low to perturb the inner Oort cloud comets to produce a death shower.

The passage of a dense IC or GMC at a distance several times its radius would produce a much weaker tidal field than during a penetrating encounter since the tidal field falls as $1 / \mathrm{r}^{3}$. Therefore the relatively frequent distant passages of GMC's discussed in Ref 5 cannot produce comet showers.

If, contrary to the estimates of Hills, ${ }^{12}$ the density of comets in the inner Oort cloud increases very rapidly with binding energy, then a death shower could possibly be produced when $a_{r} \approx 10^{4} \mathrm{AU}$. Even then, a cloud with $\mathrm{N}>10^{3}$ (Lynds class $\mathrm{j}=4$ ) would be required, and the frequency of encounters would be about one in $4.5 \times 10^{8} \mathrm{yrs}$, only $1 / 10$ of the rate of showers with the same $a_{s}$ which are induced by passing stars (see Fig 1 ). Then the rate of star induced death showers would be comparable to the rate of periodic mass extintion events, practically eliminating the need to invoke perturbations by interstellar clouds, or by a companion star, to produce several death showers per $10^{8}$ years, although the star induced showers would occur at random intervals. We conclude that encounters with dense interstellar clouds or with GMCs cannot be the reason for mass extinctions at $\approx 30 \mathrm{Myr}$ intervals ${ }^{2}$ if caused by earth impacts from intense comet showers. 
References:

1. Rampino, M.R. and Stothers, R. B. Nature 308, 709-712 (1984).

2. Raup, D. M. and Sepkoski, J. J. Proc. natn. Acad. Sci. U. S. A. 81, 801-805 (1984).

3. Stothers, R. B. Nature 311, 17 (1984).

4. Stigler, S. M. Nature 313, 159 (1985).

5. Thaddeus, P. and Chanan, G.A. Nature 314, 73-75 (1985).

6. Muller, R. A. Lawrence Berkeley Lab. Preprint LBL-20438 (1985)

7. Rampino, M.R. and Stothers, R. B. Nature 313, 159-160 (1984); Stothers, R. B. Nature 317, 338-341 (1984)

8. Oort, J. H. Bull. Astron. Inst. Neth. 11, 91-110 (1950).

9. Everhart, E. Astron. J. 73, 1039-1052 (1968); Everhart, E. and Raghavan, N. Astron. J. 75, 258-272 (1970); Marsden, B. G. and Sekanina, Z. Astron. J. 78, 1118-1124 (1973).

Weissman, P.R. IAU Colloquium 83 : Dynamics of Comets, Rome, Italy, June 1984.

10. Weissman, P.R. Astron. Astrophys. 85, 191-196 (1980)

11. Morris, D. E. and Muller, R. A. Lawrence Berkeley Lab. Preprint LBL-18942Rev2 (1985)

12. Hills, J.G. Astron. J. 86, 1730-1740 (1981).

13. Hills, J.G. Nature, 311, 636-638 (1984).

14. Rickman, H. Bull. Astr. Inst. Czech. 27, 92-105 (1976)

15. Talbot, R. J. and Newman, M. J. Astrophys. J. Suppl. 34, 295-308 (1977).

16. Sanders, D. B., Scoville, N. Z. and Solomon, P. M. Astrophys. J. 289, 373-387 (1985); 276, 182-203 (1984)

17. Blitz, L., and Shu, F.H. Astrophys. J. 238, 148-157 (1980). 


\section{Figure Caption}

Figure 1. A Comet shower will be produced when comets in the inner Oort cloud are brought into earth crossing orbits by a perturbing star or interstellar cloud (IC). The boundary between the outer Oort cloud and the inner cloud is near semi-major axes $a \approx$ $2.3 \times 10^{4} \mathrm{AU}$ (marked by the dashed vertical line). The semi-major axis $\mathrm{a}_{\mathrm{S}}$ of the smallest comet orbit which can be perturbed into an earth crossing orbit by a given class of IC is indicated, along with the cumulative rate of encounters with all classes of ICs which give smaller $\mathrm{a}_{\mathrm{S}}$. The corresponding mean time between encounters $\tau$ is also given. It is much longer than 30 Myrs, the interval between mass extinctions. The solid line gives the rate of stellar encounters which will bring in comets from a given $\mathrm{a}_{\mathbf{s}}$, after Hills. ${ }^{12}$ This rate is several times greater than that due to ICs, so passing stars induce many more comet showers than do ICs. Reduction of $\mathrm{a}_{\mathrm{s}}$ below $4 \times 10^{3} \mathrm{AU}$ is required to produce an intense "death shower" causing earth impacts. ${ }^{13}$ ICs are not dense enough to accomplish this. 


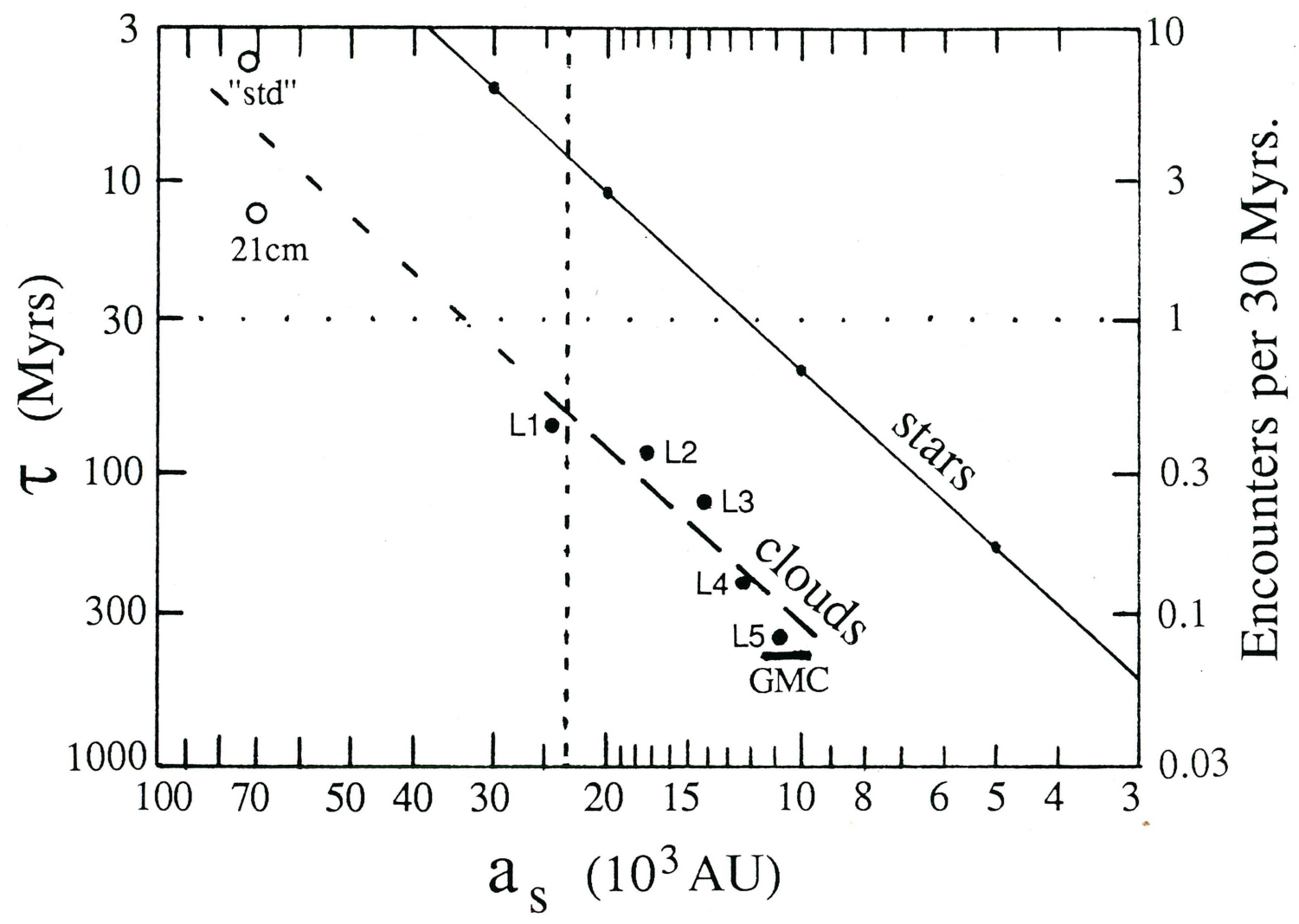

